Hitoshi Sakuraba - Fumiko Matsuzawa - Sei-ichi Aikawa

Hirofumi Doi • Masaharu Kotani • Hou Lin

Kousaku Ohno • Akemi Tanaka • Hideo Yamada

Ei-ichiro Uyama

\title{
Molecular and structural studies of the GM2 gangliosidosis 0 variant
}

Received: November 26, 2001 / Accepted: January 25, 2002

\begin{abstract}
To determine the molecular basis of the GM2 gangliosidosis 0 variant, we constructed a threedimensional structure of the human $\beta$-hexosaminidase $\beta$ subunit by homology modeling. It is composed of two domains, domains I and II, and has three disulfide bonds. C534 is located on an extra helix in domain II and forms a disulfide bond with C551. The extra helix is structurally located near domain I. C534Y, identified in a patient with the infantile form of the disease, was deduced to cause disruption of the disulfide bond, which results in a large conformational change of the extra helix, stabilizing the two domains. The drastic change in the protein structure results in a deficiency of the mature $\beta$-subunit, and deficient activities of $\beta$-hexosaminidases $A(\alpha \beta)$ and $B(\beta \beta)$, followed by abundant accumulation of GM2 ganglioside in the patient's cells. R505 is located on the eighth helix of domain II. $\mathrm{R} 505 \mathrm{Q}$, found in a patient with the chronic form of the disease, is predicted to influence the surface structure of the $\beta$-subunit, although it does not affect the active site. The
\end{abstract}

H. Sakuraba $(\bowtie) \cdot$ M. Kotani

Department of Clinical Genetics, The Tokyo Metropolitan Institute of Medical Science, Tokyo Metropolitan Organization for Medical Research, 3-18-22 Honkomagome, Bunkyo-ku, Tokyo 113-8613, Japan

Tel. +81-3-3823-2101; Fax +81-3-3823-6008

e-mail: sakuraba@rinshoken.or.jp

F. Matsuzawa $\cdot$ S. Aikawa $\cdot$ H. Doi

Celestar Lexico-Sciences, Inc., Chiba, Japan

H. Lin $\cdot$ K. Ohno

Department of Neurobiology, School of Life Sciences, Tottori

University Faculty of Medicine, Yonago, Japan

A. Tanaka

Department of Pediatrics, Osaka City University Graduate School of Medicine, Osaka, Japan

H. Yamada

Department of Pediatrics, Tokyo Metropolitan Komagome Hospital, Tokyo, Japan

E. Uyama

Department of Neurology, Kumamoto University Graduate School

of Medicine, Kumamoto, Japan amino acid substitution causes a partial processing defect and decreased enzyme activities, which result in moderate accumulation of GM2 ganglioside in the patient's cells. The structural defects well reflect biochemical and phenotypic abnormalities of the disease.

Key words GM2 gangliosidosis - Sandhoff disease · $\beta$ Hexosaminidase · Homology modeling - GM2 ganglioside

\section{Introduction}

$\beta$-Hexosaminidase (Hex; EC 3. 2. 1. 52) is a glycosyl hydrolase that catalyzes the hydrolysis of terminal $\beta-1,4$ linked $N$-acetylhexosamine residues at the nonreducing ends of oligosaccharides and their conjugates. In humans, there are two major Hex isozymes: $\beta$-hexosaminidase A (Hex A; $\alpha \beta)$, a heterodimer of subunits $\alpha$ and $\beta$, and $\beta$-hexosaminidase $B$ (Hex $B ; \beta \beta)$, a homodimer of $\beta$-subunits. Both isozymes catalyze glycoproteins, glycosaminoglycans, and glycolipids, although only Hex A can catalyze GM2 ganglioside, and GM2 activator is essential for the degradation of GM2 ganglioside in vivo. The Hex $\alpha$-subunit, $\beta$-subunit, and GM2 activator are encoded by $H E X A, H E X B$, and $G M 2 A$, respectively. Mutations in any of the three genes lead to a defect of GM2 ganglioside degradation, which causes GM2 gangliosidoses including the $\mathrm{B}, 0$, and $\mathrm{AB}$ variants, resulting from defects of $H E X A, H E X B$, and $G M 2 A$, respectively (Gravel et al. 2001).

The GM2 gangliosidosis 0 variant (McKusick 268800) is a rare autosomal recessive genetic disorder caused by mutations of $H E X B$ on chromosome 5q13. It involves both Hex A and Hex B deficiencies, and causes accumulation of GM2 ganglioside, especially in the central nervous system. The clinical phenotypes vary widely from the severe infantile form (classical Sandhoff disease), which is of early onset and fatal before the age of 4 years, to the late onset and less severe form, which allows survival into childhood or adulthood; subacute and chronic forms (atypical Sandhoff disease) also occur (Gravel et al. 2001). 
So far, about 20 mutations, including a few missense ones associated with the GM2 gangliosidosis 0 variant, have been described (Gravel et al. 2001). However, molecular structural studies on the defective Hex $\beta$-subunit have not been performed, although they are very important for clarifying the pathogenesis of the disease.

Glycosyl hydrolases have been classified into 77 families based on amino acid sequence similarity, and Hex is included in family 20 glycosyl hydrolase (Davies and Henrissat 1995). In 1996, Tews et al. (1996) refined the structure of chitobiase from Serratia marcescens, which belongs to family 20, and the data were deposited in the Protein Data Bank (PDB; Brookhaven National Laboratory, Upton, NY, USA) (Sussman et al. 1988). They also tried to model the homologous human Hex and clarify a structural rationale as to the pathogenic mutations underlying GM2 gangliosidoses. However, the target of the analysis was restricted to the predicted catalytic region because of the low sequence identity between $S$. marcescens chitobiase and human Hex. The whole structure of human wild-type Hex and the influence of missense mutations associated with GM2 gangliosidoses on the structure have been obscure. Recently, Mark et al. (2001) determined the crystal structure of Hex from Streptomyces plicatus.

In this study, we first constructed a whole structural model of the human wild-type Hex $\beta$-subunit by homology modeling and then characterized the molecular defects in Hex $\beta$-subunit caused by missense mutations identified in our patients with infantile and chronic forms of the GM2 gangliosidosis 0 variant.

\section{Patients and methods}

\section{Patients}

Patient 1. Clinical and biochemical data for the patient with the infantile form of the disease (classical Sandhoff disease) were described previously (Kuroki et al. 1995). Briefly, the patient was a 1-year-old girl with consanguineous Japanese parents. Progressive muscular weakness, hypotonia, loss of motor skills, mental retardation, and startle reaction to loud sound were observed from 6 months of age. She developed myoclonic attacks at the age of 1 year, and neurologic and ophthalmoscopic evaluations revealed a defect of the pyramidal tract and cherry red spots, respectively. Hex A and Hex B activities were both decreased to below $3 \%$ of the normal control levels, and oligosacchariduria was found. Gene analysis revealed that she was homozygous for C534Y. The numbering was performed according to Neote et al. (1988), although it had been reported to be C522Y according to Proia (1988) in the previous report.

Patient 2. Patient 2 with the chronic form of the GM2 gangliosidosis 0 variant was also previously described (Hara et al. 1998). Briefly, the patient was a 31-year-old man of Japanese origin, whose parents are consanguineous. Deterioration of handwriting, speaking, and thinking was noticed at the age of 25 years. Neurologic evaluation revealed spinocerebellar degeneration. Neither cherry-red spots nor corneal opacities were present, but marked cerebellar atrophy was observed on magnetic resonance imaging. The activities of Hex A and Hex B were $15 \%$ and $6 \%$ of the control values, respectively. Oligosacchariduria was not found. Gene analysis revealed that the patient was homozygous for R505Q, which was observed in cis with I207V. I207V had been reported to be a disease-causing mutation (Banerjee et al. 1991, 1994), but recent reports (Hara et al. 1998; Redonnet-Vernhet et al. 1996) have ruled this out, and it is now thought to be a neutral polymorphism.

The study was approved by the Ethical Committee of our institutions and informed consent was obtained from each subject or family.

Structural modeling of human wild-type and mutant Hex $\beta$-subunits

Structural models of the human wild-type Hex $\beta$-subunit and its mutants (C534Y, R505Q, I207V, and R505Q in cis with I207V) were built using molecular modeling software, SYBYL/COMPOSER and BIOPOLYMER (TRIPOS, Mountain View, CA, USA). As templates, the structures of homologous Hex from S. plicatus (PDB code; 1HP4) and chitobiase from $S$. marcescens (PDB code; 1QBA) were used. The template structures were superimposed based on the homology to determine structurally conserved regions (SCRs). Sequence alignment of the human wild-type Hex $\beta$ subunit and the template proteins was performed using Clustal W1.7 (Thompson et al. 1994) and adjusted manually to optimize the sequences of SCRs. A backbone of the human wild-type Hex $\beta$-subunit corresponding to each SCR was built by fitting a fragment from one of the templates, and the rule-based procedure was used to determine the side-chain conformations (Sutcliffe et al. 1987). Loop regions were constructed with fragments selected from the protein substructure database (Jones and Thirup 1986; Claessens et al. 1989) and with fragments generated by a loop generation method (Shenkin et al. 1987; Fine et al. 1986). All hydrogen atoms were added to each residue of the molecule according to the biopolymer dictionary (SYBYL Biopolymer Modeling Manual Ver. 6.7; TRIPOS). The energy minimization procedure was performed to optimize conformations and side-chain rotamers. The mutant models were built in the same way as for the wild type, but based on the primary structure with an amino acid replacement. To evaluate the influence of the replacement on the model structure, we superimposed the mutant model on the wild-type model based on the $\mathrm{C}^{\alpha}$ atoms by a leastsquare-mean fitting method (Kabsh 1976, 1978). We defined the structure as being influenced by the amino acid replacement when the position of an atom of the mutant differed from that of the wild type by more than the total root-mean-square distance value.

\section{Cell culture}

Cultured skin fibroblasts from Patient 1, Patient 2, normal subjects, an infantile form of GM2 gangliosidosis B variant 
(Tay-Sachs disease) patient, and a GM2 gangliosidosis AB variant (GM2 activator deficiency) patient were established and maintained in our laboratory. The cells were cultured in Ham's F-10 medium supplemented with $10 \%$ fetal calf serum and antibiotics at $37^{\circ} \mathrm{C}$ in a humidified incubator flushed continuously with a $5 \% \mathrm{CO}_{2}-95 \%$ air mixture.

\section{Western blot analysis of Hex $\alpha$ - and $\beta$-subunits}

The sizes and amounts of Hex $\alpha$ - and $\beta$-subunits in cultured fibroblasts were determined by Western blot analysis with rabbit polyclonal anti-Hex A antibodies (Izumi et al. 1988), which recognize both Hex $\alpha$ - and $\beta$-subunits, as previously reported (Ichisaka et al. 1998). Briefly, fibroblasts were harvested with a rubber policeman. Proteins were extracted with RIPA buffer $(50 \mathrm{mM}$ Tris- $\mathrm{HCl}$ at $\mathrm{pH} 7.2,150 \mathrm{mM}$ $\mathrm{NaCl}, 1 \%$ NP-40, 1\% deoxycholic acid, $0.05 \%$ sodium dodecylsulfate), and then centrifuged at $12,000 \mathrm{~g}$ for $10 \mathrm{~min}$ at $4^{\circ} \mathrm{C}$. Aliquots of the resultant supernatants were denatured, and then subjected to sodium dodecylsulfatepolyacrylamide gel electrophoresis. Thereafter, proteins were transferred to Immobilon $\mathrm{P}$ membranes (Millipore, Bedford, MA, USA), and visualized by immunostaining using anti-Hex $\mathrm{A}$ antibodies and an ECL system (Amersham Pharmacia Biotech, Buckinghamshire, UK).

Immunocytochemical analysis of intracellular GM2 ganglioside

To examine accumulation and localization of GM2 ganglioside in cells, we performed immunocytochemical analyses by double staining with a monoclonal anti-GM2 ganglioside antibody (Ig M isotype) (Kotani et al. 1992) and affinitypurified goat polyclonal antibodies (Ig G isotype; Santa Cruz Biochemistry, Santa Cruz, CA, USA) against lysosome-associated membrane protein-2 (LAMP-2). The cultured fibroblasts grown on Lab-Tek chamber slides (Nunc, Naperville, IL, USA) were fixed with ice-cold $2 \%$ paraformaldehyde in phosphate-buffered saline (PBS), $\mathrm{pH}$ 7.4 , for $10 \mathrm{~min}$, followed by blocking with $5 \%$ bovine serum albumin in PBS for $1 \mathrm{~h}$. The cells were incubated for $1 \mathrm{~h}$ with the anti-GM2 ganglioside and anti-LAMP-2 antibodies (1:100 diluted). After washing, they were reacted for $1 \mathrm{~h}$ with a fluorescein isothiocyanate-conjugated goat anti-mouse $\operatorname{IgM~} \mathrm{F}\left(\mathrm{ab}^{\prime}\right)_{2}$ (1:100 diluted; Jackson Immuno Research, West Grove, PA, USA) and a rhodamineconjugated donkey anti-goat $\operatorname{IgG~} \mathrm{F}\left(\mathrm{ab}^{\prime}\right)_{2}(1: 100$ diluted; Jackson ImmunoResearch). The stained cells were examined under a microscope (Axiovert $100 \mathrm{M}$; Carl Zeiss, Oberkochen, Germany) equipped with a confocal laser scanning imaging system (LSM510; Carl Zeiss).

\section{Results}

Structural modeling of human wild-type and mutant Hex $\beta$-subunits

According to the sequence alignment, we built a model of the human Hex $\beta$-subunit without the N-terminal 42 residues corresponding to the signal peptide. The structure corresponding to the C-terminal four residues does not exist in template structures. The model was, thus, composed of the 510 amino acids from residue 43 to terminal residue 552. The amino acid identities with the aligned fragment of $S$. plicatus Hex and that of $S$. marcescens chitobiase were $22.7 \%$ and $18.4 \%$, respectively. The modeled structure of the human Hex $\beta$-subunit is shown in Fig. 1. The human Hex $\beta$-subunit has two domains (domains I and II), which are similar to Hex from S. plicatus and two (domains II and III) of four domains in chitobiase from $S$. marcescens. In the human Hex $\beta$-subunit, domain I (residues 43-199) has an $\alpha / \beta$ topology consisting of a seven-stranded antiparallel $\beta$ sheet. Domain II (residues 200-552) is folded into a $(\beta / \alpha)_{8^{-}}$ barrel with the predicted catalytic triad (E355, H294, and D240) residing at the C termini of the eight strands of the barrel. Different from the case of $S$. plicatus Hex, the modeled domain II of the human Hex $\beta$-subunit has another long loop, loop 4, which extends from the $\mathrm{C}$ terminus of strand $\beta 4$, in addition to the three long loops (loop 2, loop 3 , and loop 7) found in S. plicatus Hex. From the energy minimization, three disulfide bonds (C91-C137, C309-C360, and C534-C551) and a free cysteine (C487) were predicted, and this result is the same as that recently reported by Schuette et al. (2001), who identified the disulfide bond structure of the enzyme biochemically. The first disulfide bond (C91-C137) is located in domain I, the second disulfide bond between C309 on loop 3 and C360 on loop 4 in domain II, and the third (C534-C551) on the extra helix in domain II. These three disulfide bonds are not observed in the corresponding locations of $S$. plicatus Hex and $S$. marcescens chitobiase. The model indicates that amino acid residues comprising an active site and hydrophobic core are conserved in human Hex $\beta$-subunit. The catalytic triad (E314, H250, and D191) found in S. plicatus Hex is conserved in the human Hex $\beta$-subunit (E355, H294, and D240, respectively). Residues D354, Y450, D191, E491, and W408 identified in the human Hex $\beta$-subunit are homologous to those responsible for the binding of a substrate in S. plicatus Hex. Residues W405, W424, and W489, which form a hydrophobic core for the binding of a substrate, are also conserved. In S. plicatus Hex, V276 on loop 3 forms the hydrophobic side of the +1 subsite with W408 on loop 7. However, it is not conserved in the human Hex $\beta$-subunit.

To reveal the influence of the mutations, we constructed the mutant structures and then compared them with the wild type. C534 is located on an extra helix in domain II and forms a disulfide bond with C551 (Fig. 1). The extra helix is structurally located near domain I. Substitution of C534 to $\mathrm{Y}$ results in disruption of the disulfide bond, and thereby 
Fig. 1A,B. Three-dimensional structure of the human $\beta$ hexosaminidase $\beta$-subunit. A Side view of the human $\beta$-hexosaminidase $\beta$-subunit. B Front view of the protein. The secondary structures are shown as tube and ribbon drawings; $\alpha$-helix (magenta), $\beta$-sheet (yellow), and coil (blue). Roman numerals indicate domains. The residue positions of cysteines forming disulfide bonds (C91-C137, C309C360, and C534-C551) and residues comprising the catalytic triad (D240, H294, and E355) are indicated by arabic numerals. The residue positions of amino acid substitutions (C534, R505, and I207) are presented as a spacefilling model. Domain I has an $\alpha /$ $\beta$ topology consisting of a sevenstranded antiparallel $\beta$-sheet. Domain II has a $(\beta / \alpha)_{8}$-barrel structure
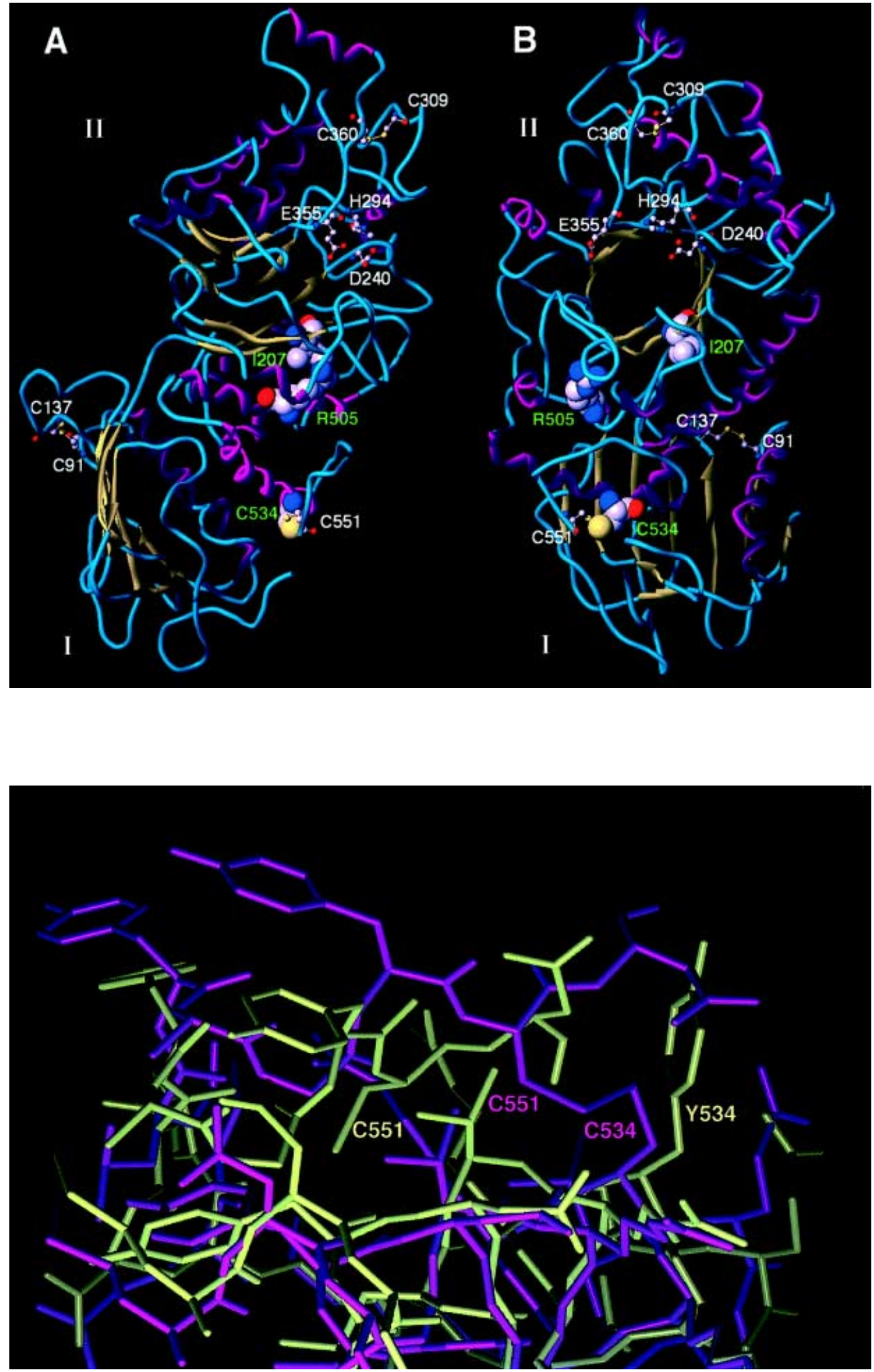

Fig. 2. Conformational changes caused by C534Y. Side views of the residues around C534 (wild type) and Y534 (mutant) are shown. The view is rotated $150^{\circ}$ in an anticlockwise direction relative to Fig. 1A. The residues of the wild type and mutant are colored magenta and yellow, respectively causes a large conformational change of the extra helix, stabilizing domains I and II (Fig. 2).

R505 is located on the eighth helix of domain II (Fig. 1). Substitution of R505 to Q results in a conformational change of the surface region, although it does not interfere with the active site (Fig. 3a). The conformational change caused by R505Q is smaller than that caused by C534Y.

I207 is located on the first strand of domain II (Fig. 1). Substitution of $\mathrm{I} 207$ to $\mathrm{V}$ does not have any significant influence on the structure (Fig. 3B). 


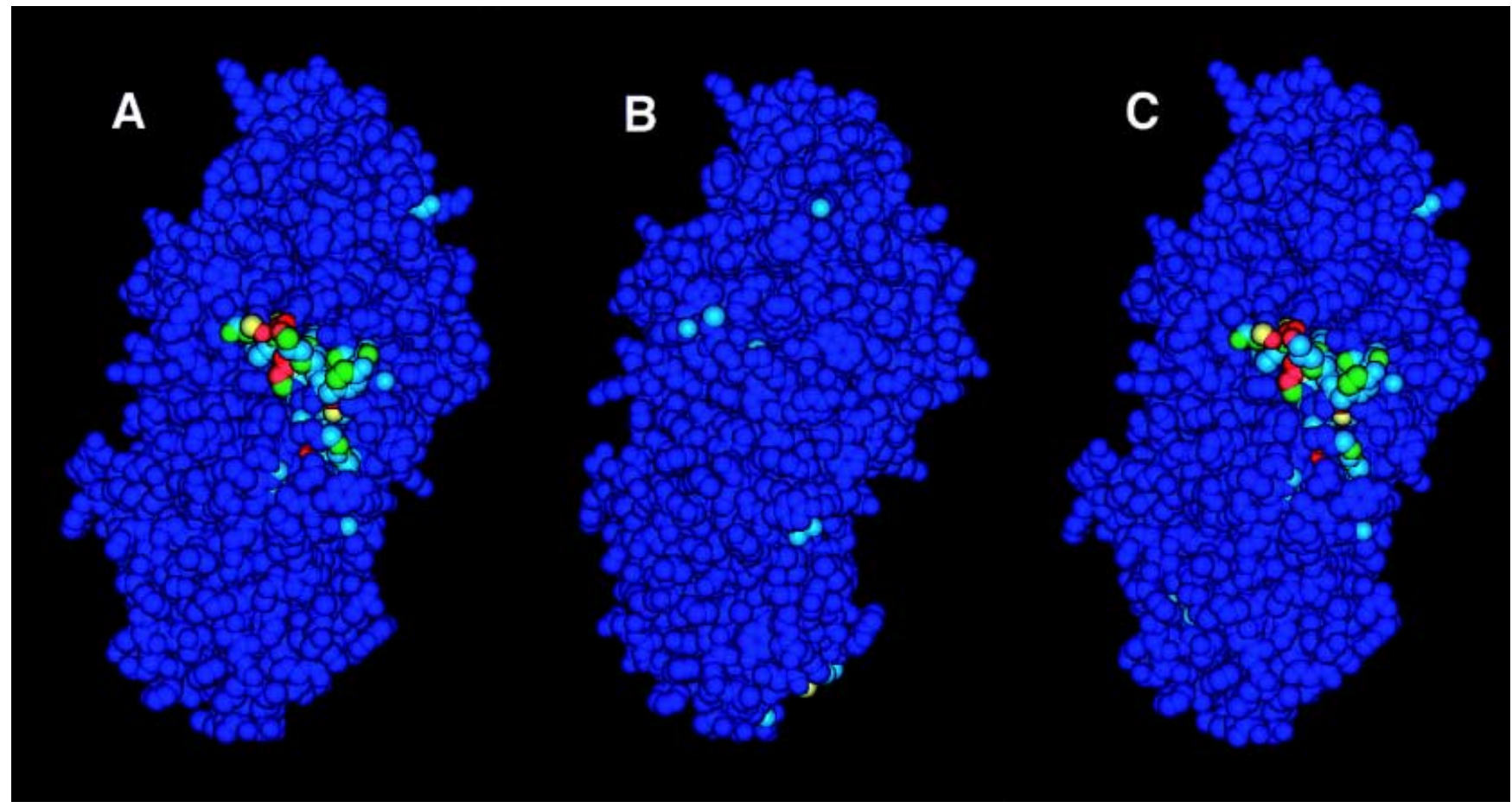

Fig. 3A-C. Structural changes of the $\beta$-subunit caused by R505Q, I207V, and R505Q in cis with I207V. The influence of amino acid substitutions of R505Q, I207V, and R505Q in cis with I207V is shown in $\mathbf{A}, \mathbf{B}$, and $\mathbf{C}$, respectively. Each deduced mutant structure is shown as a space-filling model. After superimposing the mutant and wild-type structures, each atom was colored according to the distance between the atom in the mutant and the corresponding atom in the wild-type structure. The colors of the atoms show the distance ranges, as follows: blue $<0.3 \AA, 0.3 \AA \leqq$ cyan $<0.6 \AA, 0.6 \AA$ green $<0.9 \AA, 0.9 \AA \leqq$ yellow $<1.2 \AA, 1.2 \AA \leqq$ orange $<1.5 \AA$, and red $\geqq 1.5 \AA$
R505Q in cis with I207V results in almost the same structural change as for R505Q, and no enhancement occurs with double amino acid substitutions (Fig. 3C).

\section{Western blot analysis of Hex $\alpha$ - and $\beta$-subunits}

To investigate the abnormal sizes and amounts of Hex $\alpha$ and $\beta$-subunits in cultured fibroblasts from Patients 1 and 2 , we performed Western blot analysis using total cell lysates as samples (Fig. 4). Patients 1 and 2 are homozygous for C534Y and R505Q in cis with I207V, respectively. These mutant genes are thought to reflect their products directly. We used here polyclonal anti-Hex A $(\alpha \beta)$ antibodies. These antibodies specifically recognize the precursor forms of $\alpha$ $(\alpha p)$ and $\beta(\beta p)$, the major part of the mature $\alpha$-polypeptide $(\alpha \mathrm{m})$, and the antigenic mature $\beta$-polypeptide $(\beta \mathrm{m})$ under the assay conditions used, as described previously (Ichisaka et al. 1998).

The present study revealed that the $\alpha$ - and $\beta$-subunits were synthesized in cells from both Patients 1 and 2 . However, mature $\beta$ was almost completely absent and the amount of mature $\alpha$ was also decreased in Patient 1. The decrease in mature $\alpha$ is thought to be secondary, because an $\alpha$ peptide without dimerization with $\beta$ is not stable (Gravel et al. 2001). The residual amount of mature $\alpha$ was almost the same as in the case of a patient with the infantile form of the GM2 gangliosidosis B variant (Tay-Sachs disease). In
Patient 2, mature $\beta$ and also $\alpha$ were moderately decreased, indicating residual activities of Hex A and Hex B.

Immunocytochemical analysis of intracellular GM2 ganglioside

To investigate the localization and amount of accumulated GM2 ganglioside in cells from Patients 1 and 2, we performed double staining using a specific antibody for GM2 ganglioside and polyclonal anti-LAMP-2 antibodies. The results are shown in Fig. 5. In normal fibroblasts, the antiGM2 ganglioside antibody revealed weak cytoskeletal fluorescence, which indicated the intracellularly distributed GM2 ganglioside directly bound to vimentin intermediate filaments, as described previously (Kotani et al. 1994), although the polyclonal anti-LAMP-2 antibodies could not clearly show granular staining indicating lysosomes and endosomes under the assay conditions used. In fibroblasts from Patients 1 and 2, numerous granular inclusions were observed in addition to the filamentous staining pattern. They were thought to be increased and enlarged lysosomes and endosomes with heavily accumulated GM2 ganglioside, considering the results of double staining. The fluorescence intensity in cells from Patient 1 was the same as that in cells from a patient with the GM2 gangliosidosis B variant (TaySachs disease) or a patient with the AB variant (GM2 activator deficiency). In cells from Patient 2, moderate granular 

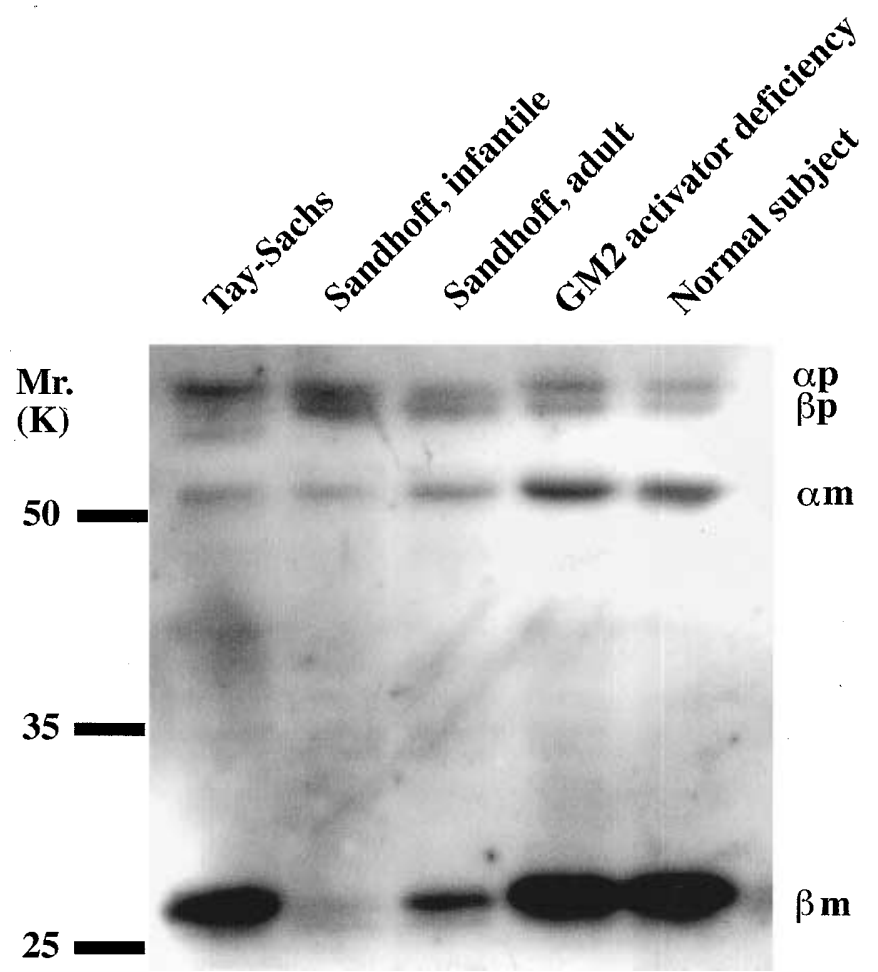

$\underset{\beta \mathbf{p}}{\alpha \mathbf{p}}$

$\alpha \mathbf{m}$

$\beta \mathbf{m}$

Fig. 4. Western blot analysis of the $\beta$-hexosaminidase $\alpha$ - and $\beta$ subunits. Cell lysates of cultured fibroblasts from a patient with the infantile form of the GM2 gangliosidosis B variant (Tay-Sachs), Patient 1 with the infantile form of the GM2 gangliosidosis 0 variant (Sandhoff, infantile), Patient 2 with the chronic form of the GM2 gangliosidosis 0 variant (Sandhoff, adult), a patient with the GM2 gangliosidosis $A B$ variant (GM2 activator deficiency), and a normal control (Normal subject) were analyzed by Western blotting using polyclonal antibodies for $\beta$-hexosaminidase $A(\alpha \beta)$. The precursors of the $\alpha$ - and $\beta$-subunits are denoted as $\alpha$ and $\beta$ p, respectively. The mature forms of the $\alpha$ - and $\beta$-subunits are denoted as $\alpha \mathrm{m}$ and $\beta \mathrm{m}$, respectively

staining was observed, indicating moderate GM2 ganglioside accumulation.

\section{Discussion}

The Hex $\alpha$ - and $\beta$-subunits undergo posttranslational processing for their localization and expression (Gravel et al. 2001; Quan et al. 1989; Hubbs et al. 1989). Their amino terminal signal peptides are removed when they are targeted to the lumen of the endoplasmic reticulum (ER). In the ER, $N$-glycosylation, disulfide-bond formation, and folding occur. Dimerization essential for catalytic activity is supposed to occur in the ER or Golgi apparatus; $\alpha \beta$ for Hex $A$ and $\beta \beta$ for Hex B. In the Golgi apparatus, further modification of sugar chains occurs and the precursor enzymes, which are fully active, are delivered to endosomes via the mannose-6-phosphate pathway. In endosomes and lysosomes, limited proteolysis results in the formation of a mature $\alpha$-subunit consisting of two peptides and a mature $\beta$ subunit consisting of three peptides (Quan et al. 1989). This processing is essential for the targeting and functions of Hex $\mathrm{A}$ and Hex B, and a quality control apparatus exists in the ER that removes improperly folded polypeptides. Therefore, the three-dimensional structures of the precursor $\alpha$ and $\beta$-subunits are very important for avoiding premature degradation and/or abnormal localization, and mutants causing improper folding are thought to cause GM2 gangliosidoses. In this study, we first constructed a model of the human Hex $\beta$-subunit using structural information on $S$. marcescens chitobiase and S. plicatus Hex.

According to the crystallographic data, S. marcescens chitobiase is folded into four domains (Tews et al. 1996), although $S$. plicatus Hex is a two-domain protein (Mark et al. 2001). The two domains of the latter have similar structures to domains II and III of the former. Despite the low sequence identity $(22.8 \%)$ between $S$. marcescens chitobiase and $S$. plicatus Hex, the topology of these domains and the active site residues are conserved in these proteins. The structure of the modeled human Hex $\beta$ subunit is basically conserved. However, there are some differences in these proteins, especially in the structures of the loops of domain II. Domain I of the human Hex $\beta$ subunit has an $\alpha / \beta$ topology, and domain II is folded into a $(\beta / \alpha)_{8}$-barrel, which may be a common structure among family 20 glycosyl hydrolases. A disulfide bond is very important for proper folding and stability of the enzyme protein. Our modeling predicted three disulfide bonds (C91-C137, C307-C360, and C534-C551) for the human Hex $\beta$-subunit. This is coincident with the recent report by Schuette et al. (2001), who determined the locations of disulfide bonds on Hex B by means of matrix-assisted laser desorption ionization mass spectrometry, and the results support the reliability of our model.

On the basis of the structural information, we tried to gain insight into the pathogenesis of the GM2 gangliosidosis 0 variant resulting from amino acid substitutions. C534 is deduced to form a disulfide bond with C551, which is essential for stabilization of domains I and II of the Hex $\beta$ subunit. C534Y can cause disruption of the disulfide bond and thereby a drastic change in the protein structure. Western blot analysis showed a deficiency of the mature $\beta$ subunit and a reduction in the amount of mature $\alpha$-subunit. The data indicate that the mutation causes a structurally unstable change in the $\beta$-subunit and/or a maturation defect. As a result, degradation of the mutant $\beta$-subunit could occur, resulting in a secondary decrease in the $\alpha$-subunit, which cannot be associated with the $\beta$-subunit in intracellular processing. The enzyme activities of both Hex A and Hex B are deficient in Patient 1, and the amount of accumulated GM2 ganglioside in her cells is high. The patient has oligosacchariduria and the severe infantile form of the disease.

On the other hand, R505Q is deduced to cause a conformational change in the surface region of the $\beta$-subunit. The deduced structural change does not influence the catalytic site but is likely to cause a folding defect. The structural change caused by R505Q is smaller than that caused by C534Y. The expressed $\beta$-subunit with R505Q was partly processed to the mature form, and showed residual enzyme 
Fig. 5. Immunocytochemical analysis of intracelluar GM2 ganglioside. Double staining of cultured fibroblasts was carried out using a monoclonal antibody for GM2 ganglioside (GM2, green) and polyclonal antibodies for LAMP2 (LAMP-2, red). Overlapping images with these two fluorescent probes are shown in yellow (GM2/LAMP-2). Normal subject, cells from a normal subject; Tay-Sachs, cells from a patient with the infantile form of the GM2 gangliosidosis B variant; Sandhoff, infantile, cells from Patient 1 with the infantile form of the GM2 gangliosidosis 0 variant; Sandhoff, adult, cells from Patient 2 with the chronic form of the GM2 gangliosidosis 0 variant; GM2 activator deficiency, cells from a patient with the GM2 gangliosidosis AB variant. Bar $50 \mu \mathrm{m}$

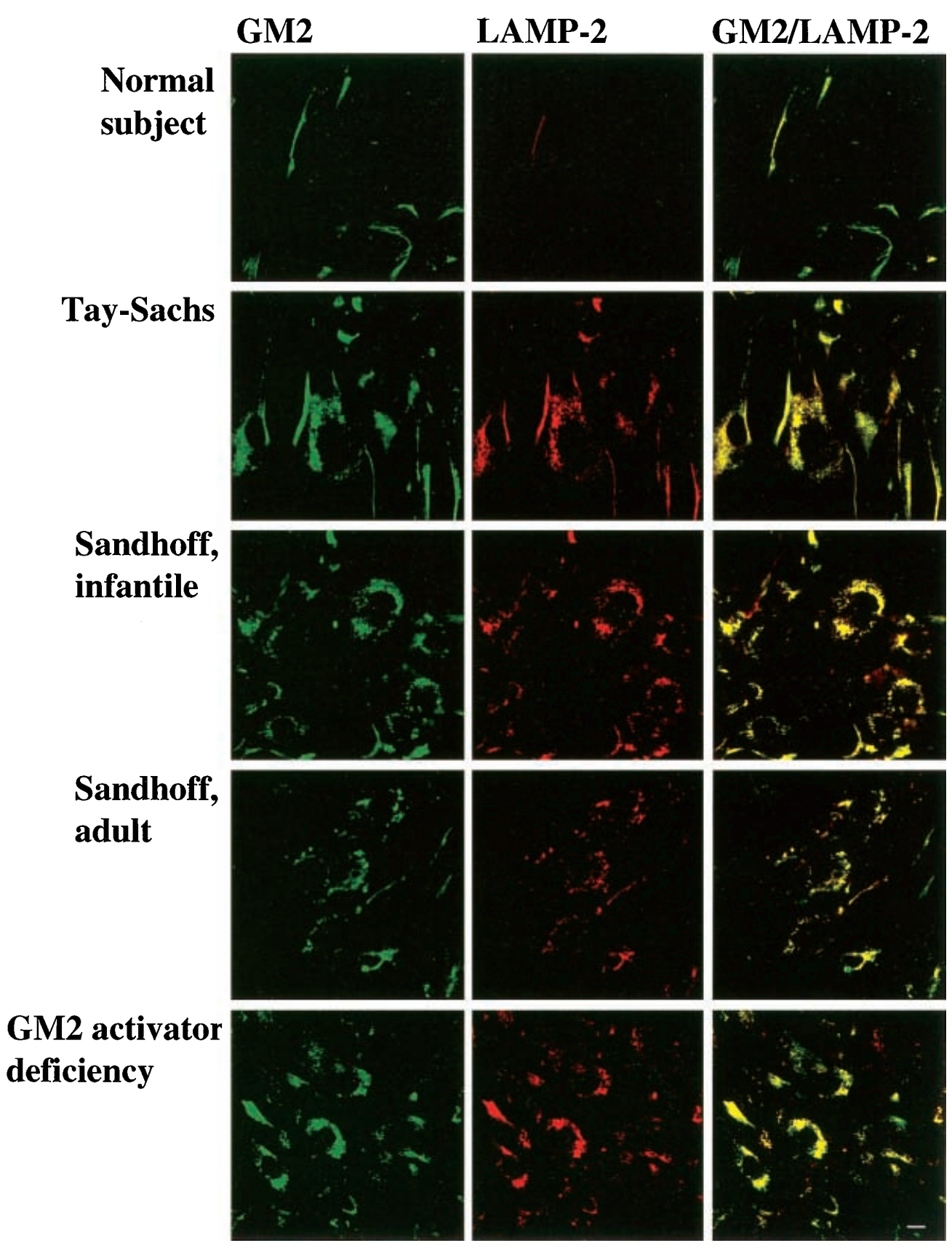

activity. Patients with this mutation show a clinically mild type of the disease. Bolhuis et al. (1993) has described a patient with $\mathrm{R} 505 \mathrm{Q}$, who developed a chronic form of the GM2 gangliosidosis 0 variant. They reported that a cDNA construct with R505Q transfected to COS cells expressed a labile form of Hex. Furthermore, Hou et al. (1998) described how P504S, which is located next to the R505 residue, decreases the level of heterodimer transport out of the ER by about $45 \%$ and lowers the heat stability, although it does not affect the $\mathrm{Km}$ values for natural or artificial substrates. We constructed a structural model of the Hex $\beta$ subunit with P504S. P504S is predicted to cause a conformational defect in the same region as that caused by R505Q (data not shown). Amino acid substitutions causing a folding defect around this lesion may result in a transport defect and the expressed mutant protein is likely to be degraded quickly.

I207V was previously reported as a disease-causing mutation (Banerjee et al. 1991; Banerjee et al. 1994), but it is now thought to be a silent polymorphism from the results of recent analyses (Hara et al. 1998; Redonnet-Vernhet et al. 1996). In this study, we constructed a mutant model with I207V and the results revealed that an amino acid substitution does not have any significant influence on the protein structure. Furthermore, the predicted change caused by $\mathrm{R} 505 \mathrm{Q}$ in cis with $\mathrm{I} 207 \mathrm{~V}$ is almost the same as in the case of 
the R505Q mutant. This indicates that I207V does not exacerbate the structural change caused by $\mathrm{R} 505 \mathrm{Q}$, and this is compatible with the clinical expression of Patient 2 who has developed a chronic and mild form of the disease.

In conclusion, we characterized the molecular basis of the GM2 gangliosidosis 0 variant with missense mutations from structural viewpoints. The data show that a molecular structural study can well explain the pathogenesis of the disease. Future determination of the crystal structures of the human Hex $\alpha$ - and $\beta$-subunits will facilitate further clarification of the complicated molecular pathologies of GM2 gangliosidoses.

Acknowledgments We wish to thank Ms. Mai Ohsawa and Seiko Yazaki for their technical assistance. This work was partly supported by grants from the Tokyo Metropolitan Government, The Japan Society for the Promotion of Science, and the Ministry of Health, Labor and Welfare of Japan.

\section{References}

Banerjee P, Siciliano L, Oliveri D, McCabe MR, Boyers MJ, Horwitz AL, Li SC, Dawson G (1991) Molecular basis of an adult form of $\beta$ hexosaminidase B deficiency with motor neuron disease. Biochem Biophys Res Commun 181:108-115

Banerjee P, Boyers MJ, Berry-Kravis E, Dawson G (1994) Preferential $\beta$-hexosaminidase (Hex) A $(\alpha \beta)$ formation in the absence of $\beta$-Hex $\mathrm{B}(\beta \beta)$ due to heterozygous point mutations present in $\beta$-Hex $\beta$ chain alleles of a motor neuron disease patient. J Biol Chem 269:4819-4826

Bolhuis PA, Ponne NJ, Bikker H, Baas F, de Jong JMBV (1993) Molecular basis of an adult form of Sandhoff disease: substitution of glutamine for arginine at position 505 of the $\beta$-chain of $\beta$ hexosaminidase results in a labile enzyme. Biochim Biophys Acta 1182:142-146

Claessens M, van Cutsem E, Lasters I, Wodak S (1989) Modeling the polypeptide backbone with "spare parts" from known protein structures. Protein Engineer 2:335-345

Davies GJ, Henrissat B (1995) Structures and mechanisms of glycosyl hydrolases. Structure 3:853-859

Fine RM, Wang H, Shenkin PS, Yarmush DL, Levinthal C (1986) Predicting antibody hypervariable loop conformations. II. Minimization and molecular dynamics studies of MCPC603 from many randomly generated loop conformations. Proteins 1:342-362

Gravel RA, Kaback MM, Proia RL, Sandhoff K, Suzuki K, Suzuki K (2001) The GM2 gangliosidoses. In: Scriver CR, Beaudet AL, Sly WS, Valle D (eds) The metabolic and molecular bases of inherited disease. 8th edn. McGraw-Hill, New York, pp 3827-3876

Hara A, Uyama E, Uchino M, Shimmoto M, Utsumi K, Itoh K, Kase R, Naito M, Sugiyama E, Taketomi T, Sukegawa K, Sakuraba H (1998) Adult Sandhoff's disease: R505Q and I207V substitutions in the $H E X B$ gene of the first Japanese case. J Neurol Sci 155:86-91

Hou Y, McInnes B, Hinek A, Karparti G, Mahuran D (1998) A Pro504 $\rightarrow$ Ser substitution in the $\beta$-subunit of $\beta$-hexosaminidase A inhibits hydrolysis of GM2 ganglioside, resulting in chronic Sandhoff disease. J Biol Chem 273:21386-21392

Hubbs M, Callahan J, Gravel R, Mahuran D (1989) The aminoterminal sequences in the pro- $\alpha$ and $-\beta$ polypeptides of human lysosomal $\beta$-hexosaminidase $\mathrm{A}$ and $\mathrm{B}$ are retained in the mature isozymes. FEBS Lett 249:316-320

Ichisaka S, Ohno K, Yuasa I, Nanba E, Sakuraba H, Suzuki Y (1998) Increased expression of $\beta$-hexosaminidase $\alpha$-chain in cultured skin fibroblasts from patients with carbohydrate-deficient glycoprotein syndrome I. Brain Dev 20:302-306

Izumi T, Fukuyama Y, Tsuji A, Yamanaka T, Hirabayashi Y, Suzuki Y (1988) GM2-gangliosidosis: B1 variant with thermostable $\beta$ hexosaminidase A and molecular analysis of the mutant enzyme. In: Salvayre R, Douste-Blazy L, Gatt S (eds) Lipid storage disorders biological and medical aspects. Plenum Press, New York, pp 237245

Jones TA, Thirup S (1986) Using known substructures in protein model building and crystallography. EMBO J 5:819-822

Kabsh W (1976) A discussion of the solution for the best rotation to relate two sets of vectors. Acta Crystallogr A32:827-828

Kabsh W (1978) A solution for the best rotation to relate two sets of vectors. Acta Crystallogr A34:922-923

Kotani M, Ozawa H, Kawashima I, Ando S, Tai T (1992) Generation of one set of monoclonal antibodies specific for a-pathway ganglioseries gangliosides. Biochim Biophys Acta 1117:97-103

Kotani M, Hosoya H, Kubo H, Itoh K, Sakuraba H, Kusubata M, Inagaki M, Yazaki K, Suzuki Y, Tai T (1994) Evidence for direct binding of intracellularly distributed ganglioside GM2 to isolated vimentin intermediate filaments in normal and Tay-Sachs disease human fibroblasts. Cell Struct Funct 19:81-87

Kuroki Y, Itoh K, Nadaoka Y, Tanaka T, Sakuraba H (1995) A novel missense mutation (C522Y) is present in the $\beta$-hexosaminidase $\beta$ subunit gene of a Japanese patient with infantile Sandhoff disease. Biochem Biophy Res Commun 212:564-571

Mark BL, Vocadlo DJ, Knapp S, Triggs-Raine BL, Withers SG, James MNG (2001) Crystallographic evidence for substrate-assisted catalysis in a bacterial $\beta$-hexosaminidase. J Biol Chem 276:10330 10337

Neote K, Bapat B, Dumbrille-Ross A, Troxel C, Schuster SM, Mahuran DJ, Gravel RA (1998) Characterization of the human $H E X B$ gene encoding lysosomal $\beta$-hexosaminidase. Genomics 3:279-286

Proia RL (1988) Gene encoding the human $\beta$-hexosaminidase $\beta$ chain: extensive homology of intron placement in the $\alpha$ - and $\beta$-chain genes. Proc Natl Acad Sci USA 85:1883-1887

Quan DVK, Proia RL, Fowler AV, Bleibaum J, Neufeld EF (1989) Proteolytic processing of the $\beta$-subunit of the lysosomal enzyme, $\beta$-hexosaminidase, in normal human fibroblasts. J Biol Chem 264:3380-3384

Redonnet-Vernhet I, Mahuran DJ, Salvayer R, Dubas F, Levade F (1996) Significance of two point mutations present in each $H E X B$ allele of patients with adult GM2 gangliosidosis (Sandhoff disease). Homozygosity for the Ile $207 \rightarrow$ Val substitution is not associated with a clinical or biochemical phenotype. Biochim Biophys Acta 1317:127-133

Schuette CG, Weisgorber J, Sandhoff K (2001) Complete analysis of the glycosylation and disulfide bond pattern of human $\beta$ hexosaminidase B by MALDI-MS. Glycobiology 11:549-556

Shenkin PS, Yarmush DL, Fine RM, Wang HJ, Levinthal C (1987) Predicting antibody hypervariable loop conformation. I. Ensembles of random conformations for ring-like structures. Biopolymers 26:2053-2085

Sussman JL, Lin D, Jiang J, Manning NO, Prilusky J, Ritter O, Abola EE (1988) Protein data bank (PDB): database of three-dimensional structural information of biological macromolecules. Acta Crystallogr D 54:1078-1084

Sutcliffe MJ, Hayes FRF, Blundell TL (1987) Knowledge based modeling of homologous proteins, part II: rules for the conformations of substituted sidechains. Protein Eng 1:385-392

Tews I, Perrakis A, Oppenheim A, Dautoer Z, Wilson KS, Vorgias CE (1996) Bacterial chitobiase structure provides insight into catalytic mechanism and the basis of Tay-Sachs disease. Nat Struct Biol 3:638-648

Thompson JD, Higgins DG, Gibson TJ (1994) CLUSTAL W: improving the sensitivity of progressive multiple sequence alignment through sequence weighting, position-specific gap penalties and weight matrix choice. Nucleic Acids Res 22:4673-4680 